\title{
Comparison of functional near-infrared spectroscopy and electrodermal activity in assessing objective versus subjective risk during risky financial decisions.
}

\author{
Holper, Lisa ; Wolf, Martin ; Tobler, Philippe N
}

\begin{abstract}
Risk is an important factor impacting financial decisions. Risk can be processed objectively, e.g. as variance across possible outcomes of a choice option or subjectively, e.g. as value of that variance to a given individual. The aim of the present study was to test the potential of functional near-infrared spectroscopy (fNIRS) in assessing these different ways of processing risk while subjects decided between either high or low risk financial options or a safe (risk-free) option. For comparison we simultaneously measured electrodermal activity (EDA), a well-established method in decision-making research and a core measure of affective processes. FNIRS showed that lateral prefrontal cortex responses to high risk were enhanced relative to low risk only in risk-seeking individuals but reduced relative to low risk in risk-averse individuals. This is in-line with individual-specific risk processing reflecting the subjective value of risk. By contrast, EDA showed enhanced responses to high risk, independent of individual risk attitude, in-line with the notion of objective risk processing. The dissociation between the two measures arose even though they overall were equally sensitive to detect individual risk-related differences and even though there was an increased, risk attitude-independent, temporal coherence between the two measures during high-risk conditions. Our results suggest that hemodynamic responses in lateral prefrontal cortex as measured by fNIRS reflect the subjective value of risk, whereas EDA may index the objective amount of risk people are presented with. The findings suggest that fNIRS could be a useful method for studying risk behavior in financial decisions.
\end{abstract}

DOI: https://doi.org/10.1016/j.neuroimage.2013.09.047

Posted at the Zurich Open Repository and Archive, University of Zurich ZORA URL: https://doi.org/10.5167/uzh-84216

Journal Article

Originally published at:

Holper, Lisa; Wolf, Martin; Tobler, Philippe N (2014). Comparison of functional near-infrared spectroscopy and electrodermal activity in assessing objective versus subjective risk during risky financial decisions. NeuroImage, 84:833-842.

DOI: https://doi.org/10.1016/j.neuroimage.2013.09.047 


\section{Comparison of functional near-infrared spectroscopy and electrodermal}

activity in assessing objective versus subjective risk during risky financial decisions

Lisa Holper ${ }^{1}$, Martin Wolf ${ }^{1}$, Philippe N. Tobler ${ }^{2}$

${ }^{1}$ Biomedical Optics Research Laboratory (BORL), Division of Neonatology, University Hospital Zurich, Frauenklinikstrasse 10, 8091 Zurich, Switzerland

${ }^{2}$ Laboratory for Social and Neural Systems Research, Department of Economics, University of Zurich, Blümlisalpstrasse 10, 8006 Zurich, Switzerland

Martin Wolf $\quad$ Martin.Wolf@usz.ch

Philippe N. Tobler phil.tobler@econ.uzh.ch

Corresponding author:

Lisa Holper, Dr.

Biomedical Optics Research Laboratory (BORL)

Division of Neonatology

University Hospital Zurich

Frauenklinikstrasse 10

8091 Zurich, Switzerland

Phone: +41 (0)44 2559792

Fax: +41(0)44 2554442

Email: holper@ini.phys.ethz.ch 


\section{Abstract}

Risk is an important factor impacting financial decisions. Risk can be processed objectively, e.g. as variance across possible outcomes of a choice option or subjectively, e.g. as value of that variance to a given individual. The aim of the present study was to test the potential of functional near-infrared spectroscopy (fNIRS) in assessing these different ways of processing risk while subjects decided between either high or low risk financial options or a safe (risk-free) option. For comparison we simultaneously measured electrodermal activity (EDA), a well-established method in decision-making research and a core measure of affective processes. FNIRS showed that lateral prefrontal cortex responses to high risk were enhanced relative to low risk only in risk-seeking individuals but reduced relative to low risk in risk-averse individuals. This is in-line with individual-specific risk processing reflecting the subjective value of risk. By contrast, EDA showed enhanced responses to high risk, independent of individual risk attitude, in-line with the notion of objective risk processing. The dissociation between the two measures arose even though they overall were equally sensitive to detect individual risk-related differences and even though there was increased, risk attitude-independent, temporal coherence between the two measures during high-risk conditions. Our results suggest that hemodynamic responses in lateral prefrontal cortex as measured by fNIRS reflect the subjective value of risk, whereas EDA may index the objective amount of risk people are presented with. The findings suggest that fNIRS could be a useful method for studying risk behavior in financial decisions.

Keywords: decision-making; neuroeconomics; risk behavior; lateral prefrontal cortex; single-trial classification; coherence analysis. 


\section{Introduction}

Risk impacts a wide variety of behaviors, including economic, financial and foraging decisions. Within the context of finance theory, risky choice options can be decomposed into statistical moments of the probability distribution of the outcomes, like the mean and the variance (mean-variance approach). In this view, the value of a choice option typically increases with the mean. Interestingly, there are substantial individual differences in how risk is processed. In risk-averse individuals, risk reduces the subjective value of a choice option whereas in risk-seeking individuals it increases value. If given a choice between a safe option and a risky option with the same expected value (EV), most people prefer the safe one, i.e. they are risk-averse. For example, when asked to choose between a box containing 10 Dollars for certain or another box with a 50/50 chance of containing 20 Dollars or being empty, most people prefer the safe option (Kahneman and Tversky, 1979). However, some prefer the risky one, i.e. they are risk-seeking. If one systematically changes the safe amount it typically turns out that also the degree of risk aversion and risk seeking varies considerably across individuals.

In the present paper, we compare two different neurophysiological methods to assess four alternatives of how individual differences in risk attitudes can manifest themselves in psychological processes and their physiological correlates. First, risk attitudes may reflect differences in the subjective valuation of risk: the more risk-averse people are, the more diminished is the subjective value of a choice option with higher risk compared to the subjective value of an option with lower risk (resulting in a negative linear relation between risk aversion and subjective value of the high risk minus the low risk option). Second, they could reflect differences in the perception of risk: the more risk-averse people are, the larger they perceive the risk of a riskier option compared to a less risky option (resulting in a positive linear relation between risk aversion and risk perception of the high risk minus the low risk option). Thirdly, they could reflect affective differences: risk-averse people may experience negative affect when exposed to risk, risk-seeking people positive affect. Accordingly, the more extreme the risk attitude, the more pronounced the affect (resulting in a curvilinear relation between risk attitude and affect induced by the high risk minus the low risk option). Lastly, it may 
well be the case that in order for valuation, perception and affect to be employed and individual differences to manifest themselves, risk first needs to be detected and processed in an objective fashion by all individuals alike (resulting in a flat relation between risk attitude and the difference between the high risk and the low risk option).

We have previously used functional magnetic resonance imaging (fMRI) and showed that the hemodynamic activity of the lateral prefrontal cortex reflects the subjective value of risk (first alternative) (Tobler et al., 2009). Specifically, the more risk-averse people are, the more lateral prefrontal activity is diminished with increasing risk, expressed in a negative linear relation between activity to high versus low risk and individual risk attitude. The first question of the present study was whether another method of measuring hemodynamic activity, i.e. functional near-infrared spectroscopy (fNIRS), could also be used for assessing this individual risk response. If so, fNIRS could then potentially be used in field studies on risky decision-making thanks to its more portable nature. So far, the method has not been widely applied in decision-making research within the laboratory. The few studies that used fNIRS in decision-related task paradigms did either not differentiate cortical signals between typical decision parameters, but reported results over whole task durations (Suhr and Hammers, 2010); or focused on non-risk-related decision aspects such as wins and losses (Cazzell et al., 2012), which do not provide insight into individual risk processing. In the present study, regarding the alternatives stated above, we expected that the hemodynamic activity of the lateral prefrontal cortex to high versus low risk measured with fNIRS shows a similar negative linear relation with risk aversion as the activity previously measured with fMRI. Such a finding would provide face-value for the use of fNIRS in financial and economic decision research.

The second question of the present study was to compare fNIRS during risky decisions with electrodermal activity (EDA). EDA offers a psychophysiological technique of measuring affective reactivity (Boucsein, 1992; Critchley et al., 2000). It has a similar temporal profile as the hemodynamic response measured by fNIRS but found much wider use in decision-making research (Figner and Murphy, 2010). Previous studies using EDA evaluating decision processing with respect to risk showed that EDA can reflect both increasing risk (Bechara et al., 1999; Studer and Clark, 2011; Yen et al., 2012) and increasing expected value (Glöckner et al., 2012; Yen et al., 2012). There are so 
far few studies that assessed simultaneously the physiological responses of fNIRS and EDA, e.g. (Combe et al., 2010; Kirilina et al., 2012; Matsuo et al., 2003; Solovey et al., 2012; Watanabe et al., 2011; Zimmermann et al., 2013), but not in decision-making research. Moreover, from a methodological point of view, it is entirely unclear whether the two methods have comparable sensitivity in detecting individual risk-related differences.

The third question of the present study was how EDA reflects individual risk attitude. So far previous research did not assess whether EDA reflects risk for example in a similar way as the hemodynamic response measured by fMRI (first alternative), or whether it reflects risk based on one of the other alternatives. In the decision-making literature EDA has typically been associated with the arousal dimension of affect, indexing its intensity (Figner and Murphy, 2010). According to this notion and the above reasoning, one would expect a curvilinear relation between risk attitude and EDA (third alternative). However, EDA has also been shown to reflect the properties of affective stimuli more objectively and irrespective of its positive or negative valence (Fowles, 1986). According to this notion and the above reasoning, one would expect a flat relation between risk attitude and EDA (fourth alternative). 


\section{Materials and Methods}

\subsection{Subjects}

Twenty healthy subjects were investigated ( 9 females, mean age ( \pm STD) $28.4 \pm 3.9$ ). All subjects were right-handed (mean laterality quotient $(\mathrm{LQ} \pm \mathrm{STD})=83.4 \pm 13.9)$ according to the Edinburgh Handedness Inventory (Oldfield, 1971). Exclusion criteria were any history of visual, neurological or psychiatric disorder or any current medication. All subjects gave written informed consent. The study was approved by the ethics committee of the Canton Zurich and performed in accordance with the Declaration of Helsinki.

\subsection{Experimental protocol}

Each subject completed a previously described risky decision-making task (Christopoulos et al., 2009; Tobler et al., 2009) and a baseline recording. The order of the conditions was counter-balanced between subjects. During the baseline recording $(120 \mathrm{sec})$, subjects were asked to fixate their eyes on a fixation cross on a black screen and to remain motionless. The decision task was implemented using the software PsychoPy (Peirce, 2007). None of the present subjects had previously taken part in experiments using this task. In each trial, subjects made choices between options of varying risk and EV displayed on the screen (Figure 1, A). A safe and a risky option appeared for $5.5 \mathrm{sec}$ on the right and left side of a fixation cross. The safe option indicated that subjects would receive the given outcome for sure $(100 \%)$, whereas the risky option indicated that the two possible outcomes were delivered with an even-chance (50\%/50\%) probability. In particular, two levels of EV were used, CHF (Swiss Francs) 30 and 60. Each of these was presented in an even-chance (50\%/50\%) low-risk and high-risk version resulting in four risky options $(15 / 45,10 / 50,40 / 80,30 / 90)$, one of which was randomly presented in each trial, together with a safe option. Thus, at an EV of CHF 30, the low-risk option offered a 50/50 chance of CHF 15 or 45 whereas the high-risk option offered CHF 10 or 50 . At the high EV level of CHF 60, the low-risk option offered CHF 40 or 80 and the high-risk CHF 30 or 90. Therefore, within each pair of conditions with the same EV, one was riskier (i.e. had higher 
variance) than the other. The safe option varied within the range of the risky option it was presented with.

Subjects were asked to choose their preferred option in each trial by considering both the safe and the risky options. Subjects had to indicate their choice between the safe and the risky option during a $2 \mathrm{sec}$ presentation of a circle around the fixation cross by pressing the arrows on a keyboard with their right hand. After the circle disappeared, the chosen option was framed for 1 sec. No outcome of subjects' choices was shown to control for the possibility that outcome-related reactivity would interfere with the choice-related responses. Finally, an inter-trial interval of variable duration occurred (10-12 sec, randomly drawn from a uniform distribution). Each session consisted of 40 trials.

At the end of the experiment, subjects received the outcome of one randomly drawn trial (in $\mathrm{CHF}$ ). If the draw resulted in a trial in which subjects had chosen a risky option, the option was played out with the toss of a coin to determine the actual outcome.

\section{[Figure 1]}

\section{3 fNIRS instrumentation and hemodynamic response modeling}

As far as they are applicable to the current context (which is cognitive and non-clinical), we followed previous recommendations (Leff et al., 2011) for fNIRS-studies, such as clear paradigm description, application of the standard sensor positioning system based on evidence of the cortical correlates described in a previous fMRI study, filtering of physiological noise, illustration of the temporal cortical hemodynamic behavior and statistical analysis of changes in all hemodynamic parameters (oxy- $\left[\mathrm{O}_{2} \mathrm{Hb}\right]$, deoxy- $[\mathrm{HHb}]$ and total hemoglobin $[\mathrm{tHb}]$ concentration) in order to improve specificity.

We used a wireless fNIRS instrument (Biocomp Research Institute, nIR system) (Figure 2). The sensor components were mounted onto a flexible printed circuit board (PCB) which, in combination with a highly flexible casing, enabled the sensor to be aligned to curved body surfaces such as the head. The optical system was a single-distance continuous-wave spectroscopy consisting of a light source with closely spaced light-emitting diodes (LEDs) emitting light at two different wavelengths $(660 \mathrm{~nm}$ and $850 \mathrm{~nm})$ and of a light detector. The distance between the source and 
detector was $3 \mathrm{~cm}$. The size of the device was $90 \mathrm{x} 40 \mathrm{~mm}$. The light intensity was sampled at $100 \mathrm{~Hz}$. The sensor was placed over the right hemisphere, covering F8 according to the international 10-20 system (Jaspers, 1958).

fNIRS data were analyzed using the model-based approach provided in NIRS-SPM (Ye et al., 2009). This toolbox for the neuroimaging suite SPM5 analyzes fNIRS data according to the general linear model. The way NIRS-SPM extracts the trial-by-trial signal amplitudes by use of a hemodynamic response function (HRF) is thought to be a reliable approximation for the concentration changes in $\left[\mathrm{O}_{2} \mathrm{Hb}\right]$ and $[\mathrm{HHb}]$ (Heilbronner and Münte, 2013). We used the wavelet minimum description length algorithm to remove systemic confounds. In estimating the temporal correlations, NIRS-SPM provides both precoloring and prewhitening methods. We employed the precoloring method (Worsley and Friston, 1995), which is according to Ye et al. (2009) more appropriate for estimating temporal correlation of NIRS data than the prewhitening method.

Dependent variables for statistical analysis were derived by subtracting the mean of the baseline $\left(\left[\mathrm{O}_{2} \mathrm{Hb}\right]_{\text {BASELINE, }}[\mathrm{HHb}]_{\text {BASELINE }}\right)$ from the mean of task-related signals $\left(\left[\mathrm{O}_{2} \mathrm{Hb}\right]_{\text {TASK }}\right.$, $\left.[\mathrm{HHb}]_{\mathrm{TASK}}\right)$, referred to as $\Delta\left[\mathrm{O}_{2} \mathrm{Hb}\right]$ and $\Delta[\mathrm{HHb}] . \Delta[\mathrm{tHb}]$, derived as the sum of $\Delta\left[\mathrm{O}_{2} \mathrm{Hb}\right]$ and $\Delta[\mathrm{HHb}]$ time series, was chosen as primary parameter of interest because it represents changes in blood volume correlated with changes in blood flow (Grubb et al., 1974) and is thought to be far less sensitive to vein contamination and therefore to provide higher spatial specificity for mapping cerebral activity compared to $\Delta\left[\mathrm{O}_{2} \mathrm{Hb}\right]$ or $\Delta[\mathrm{HHb}]$ separately (Gagnon et al., 2012). To test for statistical significance, $\Delta[\mathrm{tHb}]$ was specified within the time interval 7-12 sec after trial onset corresponding to the peak of the hemodynamic response function (Figure 3) (see section 3.2 for details on statistical analysis).

\section{[Figure 2]}

\subsection{EDA instrumentation and decomposition procedure}

To measure EDA, we used a system (Mind-Reflection, VERIM ${ }^{\circledR}$ AudioStrobe ${ }^{\circledR}$ Molinis, 16 Bit resolution, range from $10 \mathrm{k} \Omega$ to $4.5 \mathrm{M} \Omega$ ) that allowed for the acquisition of completely raw, unfiltered EDA data sampled at $100 \mathrm{~Hz}$. Two grounded flat electrodes were attached to the distal phalange of the index and middle fingers of the left, non-dominant hand prior to recording, in order to allow EDA 
levels to stabilize (Fowles et al., 1981). A custom-made MATLAB® interface was used to display and event-mark the psychophysiological data.

EDA data are usually characterized by a sequence of overlapping phasic skin conductance responses (SCRs) overlying a tonic component. For full decomposition of the skin conductance (SC) data we used the analysis software Ledalab (V3.x) (Benedek and Kaernbach, 2010) and applied continuous decomposition analysis (CDA), i.e. an extraction of the continuous phasic and tonic activity. The CDA procedure involves four steps: estimation of the tonic component, nonnegative deconvolution of phasic SC data, segmentation of driver and remainder, and reconstruction of SC data. For statistical analysis, we focused on the phasic SCRs (average phasic driver (CDA.SCR $[\mu \mathrm{u} / \mathrm{sec}]$ ); this score is thought to represent phasic activity within the response-window most accurately, but does not fall back on classic SCR amplitudes. A minimum amplitude criterion of $0.05 \mu \mathrm{S}$ was used (Levinson and Edelberg, 1985). To test for statistical significance, SCR activity was specified within the response-window 5-8 sec after trial onset corresponding to the SCR peak (Figure 3) (see section 3.2 for details on statistical analysis).

\subsection{Coherence between fNIRS and EDA}

To evaluate the temporal relationship between the fNIRS and EDA parameters, i.e. [tHb] and SCR time series, the coherence of the two signals was computed. The coherence was calculated based on the Generalized Cross S-transform (GCST) (Pinnegar and Mansinha, 2003) which allows a precise determination of the amplitude, phase and coherence associations between two signals. The coherence parameter itself incorporates information about the phase and amplitude correlations between two signals. The GCST is related to the well-known wavelet transformation techniques (Grinsted et al., 2004; Torrence and Compo, 1998) but has the advantage of having a better time-frequency resolution, a linear frequency scale and an absolutely referenced phase information. The implementation therefore allowed to select specific frequency ranges for further study. Here we tested for changes in coherence within the frequency range of $0.025-4 \mathrm{~Hz}$. Using a custom-made MATLAB® package the coherence between the two time series was calculated including the following steps: (i) calculation of the GCST, (ii) calculation of the cross wavelet power and coherence. 


\section{Results}

\subsection{Risk attitude}

Estimation of certainty equivalents (CEs) was used as formal choice-based measure of risk attitude (Luce, 2000). Using all the choices a given subject made, CEs were determined for every risky option as the points of equal preference, i.e. the safe amount for which a subject would be indifferent between the safe and the risky option. Thus, CEs quantify individual risk attitude and discriminate between risk-averse and risk-seeking individuals. Figure 1B illustrates that CEs significantly increased from low to high EV, keeping risk constant, as assessed by paired-samples t-test (confidence interval 95\%) $\left(\mathrm{t}_{19}=-9.060, \mathrm{p}<0.001\right)$. The difference in CEs for low- and high-risk options, keeping EV constant $\left(\mathrm{CE}_{\mathrm{DIFF}}=\mathrm{CE}\right.$ low-risk option $-\mathrm{CE}$ high-risk option $)$ served as index of risk attitude. Using this index, we identified risk-averse subjects $(N=14)$ indicated by a positive $\mathrm{CE}_{\mathrm{DIFF}}(>0)$ between the two options and risk-seeking subjects $(\mathrm{N}=6)$ indicated by a negative $\mathrm{CE}_{\mathrm{DIFF}}(\leq 0)$ (Figure 1C) One subject was risk-neutral, $\mathrm{CE}_{\mathrm{DIFF}}=0$. We grouped this subject together with the risk-seeking subjects because its $\mathrm{CE}_{\mathrm{DIFF}}$ was closer to their range of $\mathrm{CE}_{\mathrm{DIFF}}(-5$ to -2.5$)$ than to the range of $\mathrm{CE}_{\mathrm{DIFF}}$ of riskaverse subjects (7.5 to 15); yet, excluding the risk-neutral subject did not significantly change the results. The task identified significantly more subjects as risk-averse than risk-seeking (Fisher's Exact Test, $\chi^{2}=16.353, \mathrm{p} \leq 0.001$ ), which is in agreement with our previous findings (Christopoulos et al., 2009; Tobler et al., 2009). Together, these data show that subjects' decisions were influenced by both risk and $\mathrm{EV}$ and reveal considerable individual differences in risk attitudes.

\section{2 fNIRS and EDA responses}

We used fNIRS and EDA to assess individual differences in the processing of risk and EV. Figure 3 illustrates examples of the time course of fNIRS and EDA signals for exemplary risk-averse and riskseeking subjects. The plots represent [tHb] and SCRs in response to low- versus high-risk options with the same EV. Regarding the EDA signals, in both the risk-averse and risk-seeking example individuals the average SCRs were higher to high-risk than to low-risk options in the time interval 5-8 sec after 
option onset. Regarding the fNIRS signals, in the risk-seeking example subject the average [tHb] response was higher to high-risk than to low-risk options within the time interval 7-12sec. The inverse was true for the risk-averse example subject. Moreover, in the example risk-seeking subject the increase in $[\mathrm{tHb}]$ response to low-risk seemed to be faster than to high-risk. However, it should be noted that the changes in $[\mathrm{tHb}]$ are based on a complex interplay between the cerebral blood flow associated with a given event and the physiological reactions provoked by the brain's functional neural response, which both underlie intra-individual variability (Heinzel et al., 2013; Okada and Delpy, 2003; Okada and Delpy, 2003). The time courses of the raw signals on the single-subject level should therefore be interpreted with care.

We then performed separate ANOVAs using the two fixed factors 'Risk' (LOW RISK vs. HIGH RISK) and 'Expected Value' (LOW EV vs. HIGH EV) averaged over the time window 7-12 sec after trial onset for $\Delta[\mathrm{tHb}]$ and 5-8 sec after trial onset for SCRs. Different time windows were chosen for hemodynamic and electrodermal data in accordance with standard latencies previously described for EDA (Benedek and Kaernbach, 2010; Boucsein, 1992) and fNIRS (Huppert et al., 2006; Yücel et al., 2012) thereby taking into account the somewhat faster and steeper response of EDA compared to fNIRS (Figure 3). However, even if we used the same time windows for both methods, the findings remained the same.

\section{[Figure 3]}

\subsection{1 fNIRS responses}

To address our first question of the present study (comparison with fMRI), we evaluated the impact of risk on fNIRS signals. Over all subjects, the factor risk revealed a different effect on fNIRS signals in risk-averse and risk-seeking subjects (Figure 4, Table 1). Separate analyses for risk-averse and riskseeking subjects showed that risk had a significant, but opposite effect on hemodynamic responses in risk-averse and risk-seeking individuals. The majority of trials in risk-averse subjects revealed a decreased signal in response to the high-risk options, i.e. a negative response (Figure 3), while in approximately $30 \%$ merely a lower (but positive) response was found (note that the degree of decrease was indicative of the degree of risk aversion, see below). Importantly, an ANOVA revealed a 
significant response reduction in $\Delta[\mathrm{tHb}]$ (significant decrease in $\Delta\left[\mathrm{O}_{2} \mathrm{Hb}\right]$ and increase in $\Delta[\mathrm{HHb}]$ ) for high- compared to low-risk options in risk-averse subjects. The opposite pattern was found in riskseeking subjects, who showed a significant increase in $\Delta[\mathrm{tHb}]$ (significant increase in $\Delta\left[\mathrm{O}_{2} \mathrm{Hb}\right]$ and decrease in $\Delta[\mathrm{HHb}])$ to high- compared to low-risk options. Thus, the changes in hemodynamic responses in lateral prefrontal cortex reflected a risk attitude-dependent, impact of risk, in-line with the notion of subjective value processing.

Although of lesser importance for the present study, we also analyzed the responses to different levels of EV as these were varied as well. Over all subjects, the factor EV revealed increased hemodynamic activity with increasing EV (Figure 4, Table 1). Separate ANOVAs for risk-averse and risk-seeking subjects showed that the effect was significant for hemodynamic responses for risk-averse individuals, indicating an increase in $\Delta[\mathrm{tHb}]$ (significant increase in $\Delta\left[\mathrm{O}_{2} \mathrm{Hb}\right]$ ) to high compared to low EV. Responses in risk-seeking individuals were also higher for high than low EV, but the difference did not reach significance. To further compare risk-averse and risk-seeking subjects, we calculated for each group the difference between LOW EV and HIGH EV. A direct comparison of these differences did not reveal a stronger effect of EV in risk-averse compared to risk-seeking individuals $(\Delta[\mathrm{tHb}] \mathrm{t}=$ 0.396, $\mathrm{p}=0.692$ ). Thus these results are compatible with the notion that both groups processed EV similarly and in an increasing fashion.

\subsubsection{EDA responses}

To address our second question (comparison of fNIRS and EDA), we assessed the impact of risk on EDA. Interestingly, the SCR patterns differed from the hemodynamic ones (Figure 4, Table 1). Specifically, an ANOVA revealed that both risk-averse and risk-seeking individuals showed larger SCRs in response to high- as compared to low-risk options. These data suggest that EDA is sensitive to risk. At first sight, they seem to argue against negative or positive linear relationships between risk and responses, but curvilinear relations are still conceivable. In order to dissociate this possibility from objective risk processing, we performed more fine grained analysis by correlating individual differences with risk attitude below (see section 3.4) and found a flat relation. 
Regarding the factor EV, SCRs revealed a very similar response pattern as the hemodynamic activity (Figure 4, Table 1). Both risk-averse and risk-seeking individuals showed larger SCRs in response to high compared to low $\mathrm{EV}$, but the difference did not reach significance in risk-seeking individuals. As with the fNIRS measures, the non-significant findings for the risk-seeking group were most likely due to the small number of risk-seeking subjects in the present sample. We again calculated for each risk-averse and risk-seeking subject group the difference between LOW EV and HIGH EV. Similar to the fNIRS responses, a direct comparison did not reveal a stronger effect of EV in risk-averse compared to risk-seeking individuals (SCRs $t=0.039, \mathrm{p}=0.969$ ), supporting the notion that both groups processed EV similarly and in an increasing fashion.

\section{[Figure 4, Table 1]}

\subsection{Sensitivity analysis using single-subject classification of fNIRS and EDA data}

It could be argued that we found an effect of risk on individual differences in fNIRS but not EDA because fNIRS is more sensitive to individual differences in risk processing than EDA. We therefore performed a sensitivity comparison between fNIRS and EDA at the single-subject level. In particular, we examined whether the risk-related patterns observed in the average $\Delta[\mathrm{tHb}]$ and SCRs signals were also discriminable on the single-subject level. This was done by using Fisher's linear discriminant analysis with the class discriminatory features $\Delta[\mathrm{tHb}]$ and SCRs and the two-class classification conditions LOW RISK versus HIGH RISK by keeping EV constant. For each subject we selected the best-performing and cross-validated single-trial classification accuracy.

Results revealed an overall classification accuracy of $73.1 \%$ for fNIRS signals and $65.3 \%$ for EDA signals in discriminating responses to low- versus high-risk options (Table 2). Our data thus represented a comparable accuracy level to previous fNIRS single-trial classifications that reported accuracies ranging from 40-90\% dependent on the paradigms conducted and the classification features used (Bauernfeind et al., 2011; Holper and Wolf, 2011; Power et al., 2012). It is worth noting though that considerable inter-subject variability occurred between individual classification accuracies. However, these accuracy differences were independent of risk attitude and were consistent over both class discriminatory features. Importantly, overall comparisons using t-tests did not reveal statistical 
differences, neither between the two methods nor between risk-averse and risk-seeking subjects. Thus, regarding discrimination power, we therefore conclude that the two methods performed similarly well in discriminating individual signal patterns, suggesting that the effects of risk on hemodynamic signals are not due to higher sensitivity of fNIRS.

[Table 2]

\subsection{Correlation of fNIRS and EDA with risk attitude}

To address the third question of the present study (how EDA relates to risk attitude) we analyzed the relation of physiology and individual behavior in more detail. As reported in 3.2, risk-averse and riskseeking individuals showed differential fNIRS response patterns to risk. We assessed individual variation across all subjects for both EDA and fNIRS. In analogy to our previous fMRI study (Tobler et al., 2009), we related the physiological response differences for high versus low risk across subjects to the behavioral value differences for high versus low risk, keeping EV constant (or, in control analysis, we varied EV, keeping risk constant and related the physiological differences to risk-related value differences from behavior). Specifically, we formed risk-contrasts (HIGH RISK minus LOW RISK) for each subject. The contrasts were then used for Pearson product-moment correlation with $\mathrm{CE}_{\mathrm{DIFF}}$ as index of risk attitude (Figure 5). For $\Delta[\mathrm{tHb}]$, risk-contrasts correlated negatively with individual risk attitude $(r=-0.741, \mathrm{p} \leq 0.001)$. Conversely, no significant correlation was found for EV-contrasts $(r=0.016, \mathrm{p}=0.946$ ) or for the contrasts on SCRs (risk-contrast $r=-0.027, \mathrm{p}=0.911$; EV-contrast $r=0.022, \mathrm{p}=0.927)$. Also no curvilinear relation was detected for either measure. These findings show that individual differences in hemodynamic but not electrodermal risk responses covaried with risk attitude, such that hemodynamic responses to high risk were more reduced with higher risk aversion. Thus, the distinct patterns of a negative linear relation (fNIRS) versus a flat relation (EDA) respectively are in-line with the notion of subjective value-related risk processing in the lateral prefrontal cortex, whereas more objective, risk attitude-independent processing of risk is suggested for electrodermal responses.

[Figure 5] 


\subsection{Coherence between fNIRS and EDA}

To evaluate the temporal relationship between the fNIRS and EDA data, we computed the coherence between the $[\mathrm{tHb}]$ and SCR signals. Significant coherence values were identified in the frequency band $0.7-3 \mathrm{~Hz}$. This frequency band corresponds to the subjects' heart rate (HR). Figure 6 (Left) illustrates an example of the color-coded 2-D coherence map generated between the [tHb] and SCR time series in the HR frequency band. This plot demonstrates increases in coherence under high-risk conditions. To further validate these findings, Figure 6 (Right) illustrates the cross power of the two time series from the same measurements. This plot shows that there are no significant common changes of power in the two time series in the time-frequency domain, which could have contributed as source to the coherence increase observed between the two time series.

Within the HR frequency band, coherence values were then calculated based on the time interval 5-12 sec after trial onset. An ANOVA (Figure 4, Table 1) revealed a main effect of the factor 'Risk' (LOW RISK vs. HIGH RISK), indicating higher coherence under high-risk as compared to lowrisk conditions. Separate analysis for the two groups showed that this pattern was evident in both riskaverse and risk-seeking subjects. Importantly, there was no correlation between coherence and risk attitude. No effect was found for the fixed factor 'Expected Value' (LOW EV vs. HIGH EV). Further, no significant changes in coherence were found in the other frequency ranges tested, such as respiration rate $(0.12-0.7 \mathrm{~Hz})$, low frequency oscillations $(0.04-0.12 \mathrm{~Hz})$ and very low frequency oscillations $(0.025-0.04 \mathrm{~Hz})$.

\section{[Figure 6]}




\section{Discussion}

The present study found decreased fNIRS responses in risk-averse subjects during high- compared to low-risk options. This suggests that fNIRS can capture hemodynamic responses in lateral prefrontal cortex to risk similarly as fMRI. By using fNIRS rather than fMRI, the present findings go beyond the previous ones in that they reveal method-independence of the subjective value signals in lateral prefrontal cortex. Moreover, we find that fNIRS can detect decision-related cortical activity, thereby revealing the potential of this method in decision-making research more generally.

In addition, fNIRS responses to risk reflect its impact on subjective value, whereas EDA reflects risk more objectively independent of risk attitude, resulting respectively in negative linear versus flat relations between risk attitude and risk-related activity. Thus, similar risk attitudeindependent electrodermal responses co-occur with risk attitude-dependent hemodynamic responses. Interestingly, this dissociation arose even though the two measures were in principle similarly sensitive to reveal individual differences as assessed by single-trial classification and there was a risk attitude-independent coherence increase between hemodynamic and electrodermal activity in the heart rate frequency band.

\subsection{Comparison of fNIRS and fMRI}

The first question of the present study was how fNIRS relates to fMRI when applied to risky decisionmaking. Our fNIRS data showed a negative linear relationship between activation induced by the contrast of high versus low risk and risk aversion. This converges with our previous fMRI findings of negative linear relationships in the lateral prefrontal cortex (Christopoulos et al., 2009; Tobler et al., 2009) and the ventromedial prefrontal cortex (Tobler et al., 2007). Both of these regions have typically been implicated in processing the subjective value of choice options. Indeed, they often commonly show increased activation to high versus low value options (e.g. Plassmann et al., 2007). We corroborate and extend these fMRI findings at least for the lateral prefrontal cortex by showing that the individual impact of risk on subjective value can also be detected with fNIRS. Both modalities 
show that the injection of risk into a choice option enhances activation in risk-seeking subjects but suppresses it in risk-averse subjects. Moreover, independent of risk attitude, activation is enhanced by higher EV, as would be expected from a region processing subjective value. Thus, our data show a tight correspondence between fMRI and fNIRS data, thereby establishing fNIRS as method for studying financial decision-making.

Our experiment and data also go beyond previous applications of fNIRS to monetary reward processing (Cazzell et al., 2012) by focusing on the processing of a formally defined risk parameter in steady-state situations rather than wins and losses in ambiguous learning situations (with risk, probabilities are known, with ambiguity they are not). Here we capture risk as variance, in agreement with the standard mean-variance approach used by finance theory but higher-level risk terms, such as skewness, also affect behavior and neural activity (e.g. Burke and Tobler, 2011).

\subsection{Comparison and neurophysiological interpretation of fNIRS and EDA}

The second question of the present study was how risk-related responses compared between fNIRS and EDA. Risk-induced hemodynamic responses of the lateral prefrontal cortex showed a negative linear relation to risk attitude, which resulted in reduced responses to risk in risk-averse subjects, but increased responses in risk-seeking subjects. Interpretation of these patterns could be based on two neurophysiological concepts: activation versus deactivation in lateral prefrontal cortex and/or activation in other cerebral areas. First, the increased hemodynamic signal in risk-seeking subjects represents a 'typical' fNIRS response, whereas the reduced or decreased hemodynamic signal in riskaverse individuals can be described as 'inverse' or negative response (Strangman et al., 2002). Inverse responses may reflect what has been described as negative blood oxygenation level dependent (BOLD) response (NBR) in fMRI as an exact mirror image of the positive BOLD response (PBR) (Shmuel et al., 2002). In the current understanding PBR reflects neural excitation (excitatory potentials), or active inhibition (inhibitory potentials) (Logothetis, 2003), whereas NBR reflects less neural processing due to reduced cerebral blood flow, oxygen consumption or neuronal deactivation (Shmuel et al., 2002). In this sense, the inverse response in risk-averse subjects might be a result of a deactivation or decrease in neural activity of the lateral prefrontal cortex. Alternatively, the inverse 
hemodynamic response in risk-averse subjects may be due to simultaneous activation in other neighboring decision-related prefrontal cortical or even subcortical regions. This may have induced a kind of 'steal' effect by which blood is diverted to active sites (e.g. medial prefrontal cortex, oribitofrontal cortex) from the lateral prefrontal cortex (Harel et al., 2002). Although the underlying cortical processes remain unclear, these physiological aspects may be considered as separate or combined processes when interpreting our data.

Compared to the hemodynamic responses, EDA responses revealed a different pattern. SCR magnitudes were larger in response to high- than low-risk options (Figure $3 \& 4$, Table 1). This pattern occurred similarly in both risk-seeking and risk-averse subjects indicating that EDA reflects levels of risk, but does so irrespective of individual risk attitude. This is reasonable since, contrary to the lateral prefrontal hemodynamic responses reflecting the qualitative aspect of subjective value, the physiological components of SCRs are expressed based on the same functional origin of the skin's eccrine activity in both subject groups, i.e. reflecting a quantitative and more objective response signal (Fowles, 1986).

As recently reviewed (Figner and Murphy, 2010), it is important to note what can and cannot be assessed with skin conductance. It is well-established that SCRs covary with the sympathetic arousal dimension of affect, indexing its intensity. Contrary to this quantitative aspect, the qualitative aspects of affect, such as its valence (positive/negative, approach/avoidance) or which emotion is present (e.g. fear of risk versus joy in risk, etc.) are not reflected in EDA and have to be inferred from other sources. Note though that this would nevertheless be compatible with a curvilinear relation between risk aversion and EDA. Contrary to such a relation, however, even subjects close to risk neutrality showed a substantial and similar response to high risk as risk-averse and risk-seeking subjects. This resulted in a flat relation between EDA and risk attitude, indicative of objective risk processing. Thus, extremely risk-sensitive subjects were not generally more responsive to risk than less risk-sensitive subjects, even though this could have been conjectured based on the hemodynamic responses. Taken together, our data suggest that distinct physiological measures of risk processing reflect distinct psychological functions, which in turn are implemented in distinct (but coherent, see below) physiological systems. 


\subsection{Coherence between fNIRS and EDA}

We evaluated the temporal relationship between the hemodynamic and electrodermal responses by investigating the coherence between the $[\mathrm{tHb}]$ and SCRs time series. Coherence analyses have been used in various EEG and fMRI studies as complementary tool to investigate the dynamic relationship between the central and autonomic nervous system, in particular when a priori assumptions of linear coupling are made (Chang and Glover, 2010; Klein et al., 2006; Li et al., 2007; Müller et al., 2004).

Our results showed a significant increase in coherence specifically in the HR frequency band with increasing risk. In the following discussion we therefore focus on HR-effects. As discussed above, risk induced different fNIRS and EDA signals in the risk-averse and risk-seeking subject groups. Consequently, it could have been expected that these opposite patterns led to decreased coherence patterns. However, our calculations clearly indicated a significant coherence increase when subjects made decisions under high-risk conditions in both risk-averse and risk-seeking subjects (Figure $6 \&$ 7, Table 1). This coherence pattern might be based on the following physiological observations. Variations in HR relate to both hemodynamic responses and behavioral processes. For example, HR variations in response to arousing stimuli have been reported to follow two main patterns. First, there is an initial HR slowing, i.e. a HR deceleration, thought to express attentional orienting to motivationally-salient events through parasympathetic activity (Bradley, 2009). Second, there is a later rise in HR, i.e. a HR acceleration, signifying affective arousal through sympathetic activation (Bradley and Lang, 2007). In the decision-making literature, these two patterns have been also reported during risky decision-making (Jones et al., 2011; Studer and Clark, 2011), with HR responses being sensitive to potential risky choices, i.e. stronger HR decelerations were observed in trials with lower chances of winning (Studer and Clark, 2011).

Regarding our data, the following speculation might be considered. Based on previous literature (Studer and Clark, 2011), it could be assumed that during the presentation of high-risk options subjects' HR decelerated (before accelerating and going back to baseline). Further, based on the absence of any correlation of risk attitude with the coherence measure, we might assume that this HR decrease occurred independent of risk attitude, similar to the EDA increase. The common decrease 
in HR might in turn have induced a higher temporal synchronization (coherence increase) between the neural and the electrodermal systems during high-risk conditions. This coherence increase was found in the trial window 5-12 sec after task onset, supporting the assumption that it is linked to the initial HR deceleration, whereas the potentially following HR acceleration at the end of the trial did not result in increased coherence. This functional physiological link between the two systems could be interpreted as facilitation for achieving best decision performance under high-risk conditions. Such optimal performance may require both systems to coordinate.

This tentative interpretation is not intended to be the only one, but gives one possible explanation of how the dynamic relationship between the underlying physiological and psychological processes could be reflected in the coherence data. Future studies are required to test this and other possibilities. Assessing functional synchronization between central and peripheral parameters may also be attractive for other applications involving decision paradigms seeking to provide insight into behavioral performance and associated physiological changes.

\subsection{Relation of EDA and risk attitude}

The third question of the present study was how EDA reflects individual risk attitude. In the introduction, we considered four alternatives of how risk attitudes may manifest themselves in mental functions and their physiological correlates (negative linear, positive linear, curvilinear and flat relation). For hemodynamic fMRI responses, we have previously found response increases with risk but a flat relation with risk attitude in the middle cingulate cortex (Burke and Tobler, 2011; Christopoulos et al., 2009) and posterior parts of the lateral orbitofrontal cortex (Tobler et al., 2007). Such relationships are compatible with objective risk processing in the present study observed with electrodermal responses. Both cingulate and orbitofrontal cortex have been implicated in the processing of EDA (Dubé et al., 2009) and it is conceivable that our EDA data reflect signals generated in these regions. The extreme outcomes of risky options tend to attract more attention and thus be more salient than the safe ones of intermediate magnitude (Ogawa et al., 2013). Hence, it is conceivable that increases of both EDA and hemodynamic activity in these regions are due to increased salience of higher compared to lower risk. Further, the presently found elevation of EDA 
with higher EV is compatible with this notion as higher EV also is associated with higher salience. In any case, by showing that neither risk- nor EV-related EDA responses are related to risk attitude even though both factors increase them on average, the present study adds and goes beyond previous studies reporting increasing EDA separately with increasing EV (Glöckner et al., 2012; Yen et al., 2012) and increasing risk (Bechara et al., 1999; Studer and Clark, 2011).

We also considered positive linear and curvilinear relationships. A positive linear relationship between activation and risk aversion is compatible with risk being perceived as larger with increasing risk aversion. Such a relationship has been reported for example in the insula (Rudorf et al., 2012) and central orbitofrontal regions (Tobler et al., 2007). Although both of these regions are relatively close to the currently recorded lateral prefrontal region, we did not find a positive relation in our fNIRS data, thereby corroborating regional specificity of the present set-up (note that due to limited depth penetration fNIRS is not able to detect changes in deeper brain tissue such as the insula). Finally, curvilinear relationships may imply that more extreme risk attitudes are associated with more pronounced (positive or negative) affect elicited by risk. This notion may be compatible with the "risk as feelings" hypothesis (Slovic et al., 2004). To our knowledge, curvilinear relations remain to be investigated on the neural level.

\subsection{Conclusion}

We show that risk differently impacts lateral prefrontal hemodynamic and electrodermal decision correlates. In particular, high risk elevated hemodynamic responses only in risk-seeking subjects but suppressed them in risk-averse subjects. This coincided with a negative linear relation with risk attitude, indicative of subjective value processing. In contrast, elevated electrodermal responses to high versus low risk coincided with a flat relation to risk attitude, indicative of objective risk processing. Moreover, we report a high risk-induced coherence increase between electrodermal and hemodynamic responses that provides an additional layer of information concerning the temporal relationship between central and peripheral physiological systems involved in decision-making. Finally, our approach demonstrates the potential of fNIRS in measuring the neural correlates of processing risk as defined by financial decision theory. The successful validation of the fNIRS data 
with a previous fMRI study suggests that fNIRS has potential to quantify neural activity in more natural settings than the MRI scanner.

\section{Acknowledgement}

The authors thank all participants for taking part in this research as well as the Swiss Foundation for Grants in Biology and Medicine (SFGBM) and the Swiss National Science Foundation (Grants PASMP3_136987, PP00P1_128574, and CRSII3_141965) for financial support.

Disclosure Statement: The authors have no conflict of interest. 


\section{References}

Bauernfeind, G., Scherer, R., Pfurtscheller, G., Neuper, C., 2011. Single-trial classification of antagonistic oxyhemoglobin responses during mental arithmetic. Medical and Biological Engineering and Computing 49, 979-984.

Bechara, A., Damasio, H., Damasio, A.R., Lee, G.P., 1999. Different contributions of the human amygdala and ventromedial prefrontal cortex to decision-making. The Journal of Neuroscience 19, $5473-5481$.

Benedek, M., Kaernbach, C., 2010. Decomposition of skin conductance data by means of nonnegative deconvolution. Psychophysiology 47, 647-658.

Boucsein, W., 1992. Electrodermal activity. Plenum Press, New York, NY.

Bradley, M.M., 2009. Natural selective attention: orienting and emotion. Psychophysiology 46, 1-11.

Bradley, M.M., Lang, P., 2007. Emotion and motivation, in: Handbook of Psychophysiology. Cambridge University Press, Cambridge, MA.

Burke, C.J., Tobler, P.N., 2011. Reward skewness coding in the insula independent of probability and loss. Journal of Neurophysiology 106, $2415-2422$.

Cazzell, M., Li, L., Lin, Z., Patel, S., Liu, H., 2012. Comparison of neural correlates of risk decision making between genders: An exploratory fNIRS study of the Balloon Analogue Risk Task (BART). NeuroImage 62, 1896-1911.

Chang, C., Glover, G., 2010. Time frequency dynamics of resting-state brain connectivity measured with fMRI. NeuroImage 50, 81-98.

Christopoulos, G.I., Tobler, P.N., Bossaerts, P., Dolan, R.J., Schultz, W., 2009. Neural correlates of value, risk, and risk aversion contributing to decision making under risk. The Journal of Neuroscience 29, $12574-12583$.

Combe, E., Suzuki, K., Chao, Z., Fujii, N., 2010. Facing a threat in a 3D environment: evaluation of the impact of depth perception on the emotional state by skin conductance and near-infrared spectroscopy. Journal of Neuroscience Research 68, 3268.

Critchley, H.D., Elliott, R., Mathias, C.J., Dolan, R.J., 2000. Neural activity relating to generation and representation of galvanic skin conductance responses: A functional magnetic resonance imaging study. The Journal of Neuroscience 20, 3033 -3040.

Dubé, A., Duquette, M., Roy, M., Lepore, F., Duncan, G., Rainville, P., 2009. Brain activity associated with the electrodermal reactivity to acute heat pain. NeuroImage 45, 169-80.

Figner, B., Murphy, R., 2010. Using skin conductance in judgment and decision making research. In M. Schulte-Mecklenbeck, A. Kuehberger, \& R. Ranyard (Eds.). A handbook of process tracing methods for decision research.

Fowles, D., 1986. The eccrine system and electrodermal activity, in: Psychophysiology. Guilford Press, New York, pp. 51-96.

Fowles, D.C., Christie, M.J., Edelberg, R., Grings, W.W., Lykken, D.T., Venables, P.H., 1981. Publication recommendations for electrodermal measurements. Psychophysiology 18, 232239.

Gagnon, L., Yücel, M.A., Dehaes, M., Cooper, R.J., Perdue, K.L., Selb, J., Huppert, T.J., Hoge, R.D., Boas, D.A., 2012. Quantification of the cortical contribution to the NIRS signal over the motor cortex using concurrent NIRS-fMRI measurements. NeuroImage 59, 3933-3940.

Glöckner, A., Fiedler, S., Hochman, G., Ayal, S., Hilbig, B., 2012. Processing differences between descriptions and experience: a comparative analysis using eye-tracking and physiological measures. Frontiers in Psychology 3, 173.

Grinsted, A., Moore, J., Jevrejeva, S., 2004. Application of the cross wavelet transform and wavelet coherence to geophysical time series. Nonlinear Processes in Geophysics 11, 561-566. 
Grubb, R., Raichle, M., Eichling, J., Ter-Pogossian, M., 1974. The effects of changes in PaCO2 cerebral blood volume, blood flow, and vascular mean transit time. Stroke 5, 630 -639.

Harel, N., Lee, S., Nagaoka, T., Kim, D., Kim, S., 2002. Origin of negative blood oxygenation leveldependent fMRI signals. Journal of Cerebral Blood Flow \& Metabolism 22, 908-17.

Heilbronner, U., Münte, T., 2013. Rapid event-related near-infrared spectroscopy detects age-related qualitative changes in the neural correlates of response inhibition. NeuroImage 65, 408-15.

Heinzel, S., Haeussinger, F., Hahn, T., Ehlis, A., Plichta, M., Fallgatter, A., 2013. Variability of (functional) hemodynamics as measured with simultaneous fNIRS and fMRI during intertemporal choice. NeuroImage 71, 125-134.

Holper, L., Wolf, M., 2011. Single-trial classification of motor imagery differing in task complexity: a functional near-infrared spectroscopy study. Journal of NeuroEngineering and Rehabilitation 8,34 .

Huppert, T.J., Hoge, R.D., Diamond, S.G., Franceschini, M.A., Boas, D.A., 2006. A temporal comparison of BOLD, ASL, and NIRS hemodynamic responses to motor stimuli in adult humans. NeuroImage 29, 368-382.

Jaspers, H., 1958. The ten-twenty electrode system of the International Federation. Electroencephalography and Clinical Neurophysiology 10, 371-375.

Jones, C., Minati, L., Harrison, N.A., Ward, J., Critchley, H., 2011. Under pressure: Response urgency modulates striatal and insula activity during decision-making under risk. PLoS ONE 6, e20942.

Kahneman, D., Tversky, A., 1979. Prospect theory: An analysis of decision under risk. Econometrica 47, 263-291.

Kirilina, E., Jelzow, A., Heine, A., Niessing, M., Wabnitz, H., Brühl, R., Ittermann, B., Jacobs, A., Tachtsidis, I., 2012. The physiological origin of task-evoked systemic artefacts in functional near infrared spectroscopy. NeuroImage 61, 70-81.

Klein, A., Sauer, T., Jedynak, A., Skrandies, W., 2006. Conventional and wavelet coherence applied to sensory-evoked electrical brain activity. IEEE Trans Biomed Eng 53, 266-72.

Leff, D.R., Orihuela-Espina, F., Elwell, C.E., Athanasiou, T., Delpy, D.T., Darzi, A.W., Yang, G.-Z., 2011. Assessment of the cerebral cortex during motor task behaviours in adults: A systematic review of functional near infrared spectroscopy (fNIRS) studies. NeuroImage 54, 2922-2936.

Levinson, D.F., Edelberg, R., 1985. Scoring criteria for response latency and habituation in electrodermal research: A critique. Psychophysiology 22, 417-426.

Li, X., Yao, X., Fox, J., Jefferys, J., 2007. Interaction dynamics of neuronal oscillations analysed using wavelet transforms. Journal of Neuroscience Methods 160, 178-85.

Logothetis, N.K., 2003. MR imaging in the non-human primate: studies of function and of dynamic connectivity. Current Opinion in Neurobiology 13, 630-642.

Luce, R., 2000. Utility of gains and losses. Erlbaum, Mahwah, NJ.

Matsuo, K., Kato, T., Taneichi, K., Matsumoto, A., Ohtani, T., Hamamoto, T., Yamasue, H., Sakano, Y., Sasaki, T., Sadamatsu, M., Iwanami, A., Asukai, N., Kato, N., 2003. Activation of the prefrontal cortex to trauma-related stimuli measured by near-infrared spectroscopy in posttraumatic stress disorder due to terrorism. Psychophysiology 40, 492-500.

Müller, K., Lohmann, G., Neumann, J., Griqutsch, M., Mildner, T., von Cramon, D., 2004. Investigating the wavelet coherence phase of the BOLD signal. Journal of Magnetic Resonance Imaging 20, 145-52.

Ogawa, M., van der Meer, M., Esber, G., Cerri, D., Stalnaker, T., Schoenbaum, G., 2013. Riskresponsive orbitofrontal neurons track acquired salience. Neuron 77, 251-8.

Okada, E, Delpy, D., 2003. Near-infrared light propagation in an adult head model. I. Modeling of low-level scattering in the cerebrospinal fluid layer. Applied Optics 42, 2906-14. 
Okada, E., Delpy, D., 2003. Near-infrared ligth propagation in an adult head model. II. Effect of superficial tissue thickness on the sensitivity of the near-infrared spectroscopy signal. Applied Optics 42, 2915 - 2922.

Oldfield, R., 1971. The assessment and analysis of handedness: the Edinburgh inventory. Neuropsychologia 9, 97-113.

Peirce, J., 2007. PsychoPy - Psychophysics software in Python. Journal of Neuroscience Methods 162, 8-13.

Pinnegar, C., Mansinha, L., 2003. The S-transform with windows of arbitrary and varying shape. Geophysics 68, 381-385.

Plassmann, H., O’Doherty, J., Rangel, A., 2007. Orbitofrontal cortex encodes willingness to pay in everyday economic transactions. The Journal of Neuroscience 27, $9984-9988$.

Power, S., Kushki, A., Chau, T., 2012. Intersession consistency of single-trial classification of the prefrontal response to mental arithmetic and the no-control state by NIRS. PLoS ONE 7, e37791.

Rudorf, S., Preuschoff, K., Weber, B., 2012. Neural correlates of anticipation risk reflect risk preferences. The Journal of Neuroscience 32, 16683-16692.

Shmuel, A., Yacoub, E., Pfeuffer, J., Van de Moortele, P.-F., Adriany, G., Hu, X., Ugurbil, K., 2002. Sustained negative BOLD, blood flow and oxygen consumption Response and its coupling to the positive response in the human brain. Neuron 36, 1195-1210.

Slovic, P., Finucane, M., Peters, E., MacGregor, D., 2004. Risk as analysis and risk as feelings: Some thoughts about affect, reason, risk, and rationality. Risk Analysis 24, 311-322.

Solovey, E., Mehler, B., Reimer, B., 2012. Brain sensing with fNIRS in the car, in: Adjunct Proceedings of the 4th International Conference on Automotive User Interfaces and Interactive Vehicular Applications. Presented at the AutomotiveUI '12), Portsmouth, NH, USA.

Strangman, G., Culver, J., Thompson, J., Boas, D., 2002. Quantitative comparison of simultaneous BOLD fMRI and NIRS recordings during functional brain activation. Neuroimage 17, $719-$ 731.

Studer, B., Clark, L., 2011. Place your bets: psychophysiological correlates of decision-making under risk. Cognitive, Affective, \& Behavioral Neuroscience 11, 144-158.

Suhr, J., Hammers, D., 2010. Who Fails the Iowa Gambling Test (IGT)? Personality, Neuropsychological, and Near-Infrared Spectroscopy Findings in Healthy Young Controls. Archives of Clinical Neuropsychology 25, 293 -302.

Tobler, P.N., Christopoulos, G.I., O’Doherty, J.P., Dolan, R.J., Schultz, W., 2009. Risk-dependent reward value signal in human prefrontal cortex. Proceedings of the National Academy of Sciences 106, $7185-7190$.

Tobler, P.N., O'Doherty, J.P., Dolan, R.J., Schultz, W., 2007. Reward value coding distinct from risk attitude-related uncertainty coding in human reward systems. Journal of Neurophysiology 97, $1621-1632$.

Torrence, C., Compo, G., 1998. A practical guide to wavelet analysis. Bulletin of the American Meteorological Society 79, 61-78.

Watanabe, Y., Hosokawa, M., Sumitani, S., Yamamoto, M., Fukuda, S., Ohmori, T., 2011. Prefrontal activation during emotional experience as measured by NIRS. JAIRO 19, 49-57.

Worsley, K.J., Friston, K., 1995. Analysis of fMRI time-series revisited-again. NeuroImage 2, $173-$ 81.

Ye, J.C., Tak, S., Jang, K.E., Jung, J., Jang, J., 2009. NIRS-SPM: Statistical parametric mapping for near-infrared spectroscopy. NeuroImage 44, 428-447. 
Yen, N., Chou, I., Chung, H., Chen, K., 2012. The interaction between expected values and risk levels in a modified Iowa gambling task. Biological Psychology 91, 232-7.

Yücel, M.A., Huppert, T., Boas, D., Gagnon, L., 2012. Calibrating the BOLD signal during a motor task using an extended fusion model incorporating DOT, BOLD and ASL data. NeuroImage 61, 1268-1276.

Zimmermann, R., Marchal-Crespo, L., Edelmann, J., Lambercy, O., Fluet, M., Riener, R., Wolf, M., Gassert, R., 2013. Detection of motor execution using a hybrid fNIRS-biosignal BCI: a feasibility study. Journal of NeuroEngineering and Rehabilitation 10. 


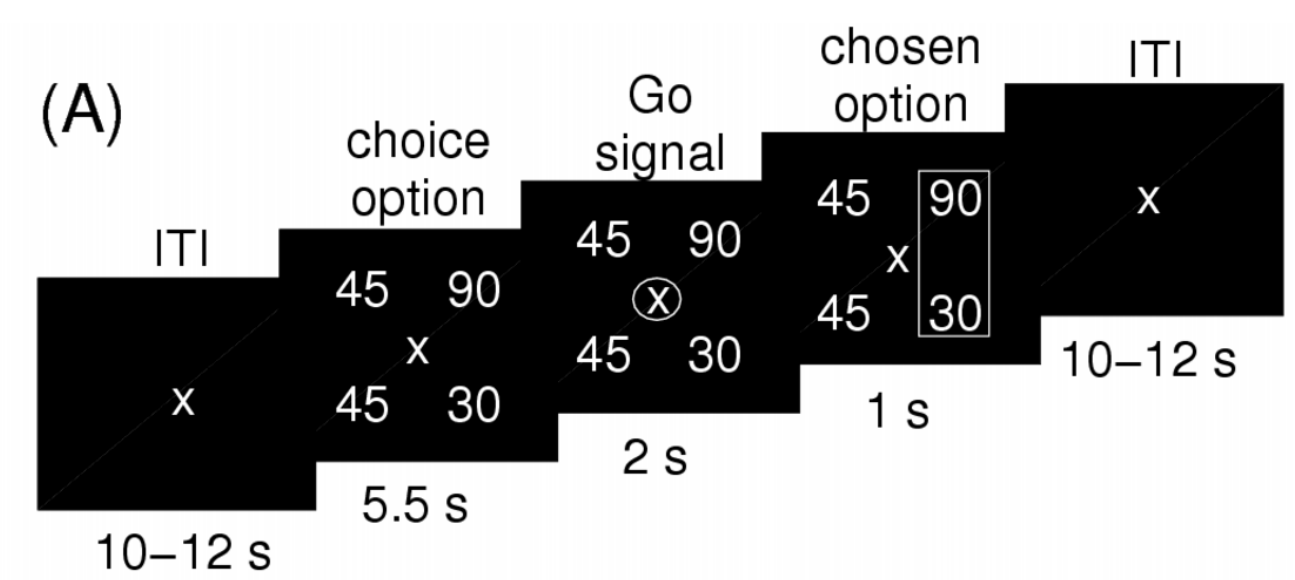

\section{(B)}

(C)
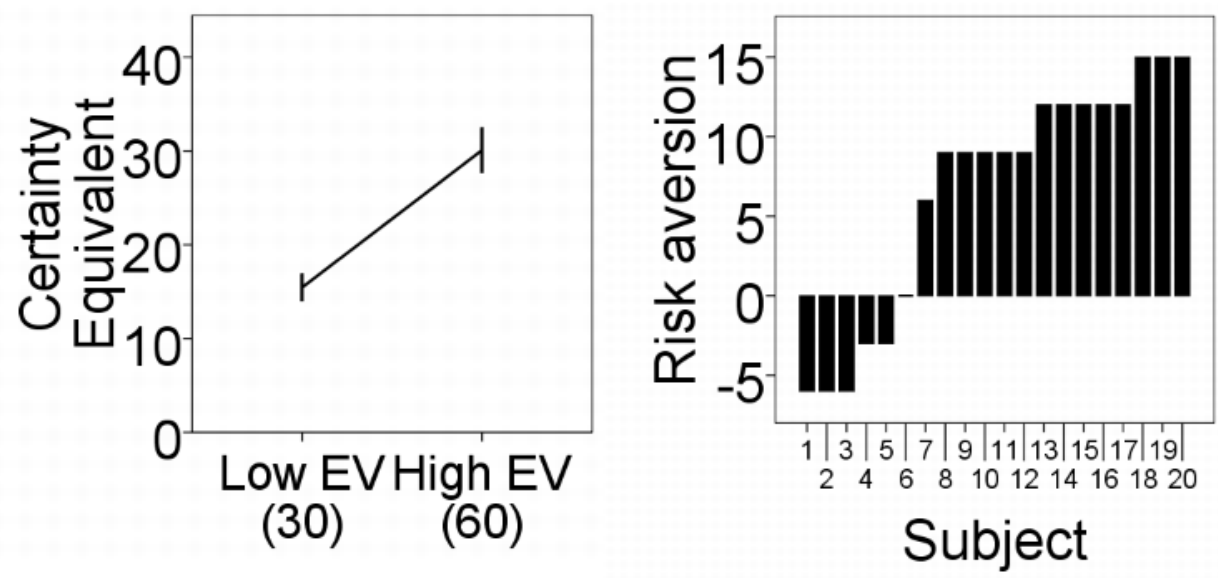

Figure 1. (A) Experimental design. Shown is the trial structure including option presentation, Go signal, chosen option and inter-trial interval (ITI). (B) Certainty equivalents. Average certainty equivalents (CEs) of low and high expected value (EV) options with same risk; error bars represent standard error of the mean (SEM). (C) Risk aversion. Risk aversion of single subjects as measured by the difference between CEs of low- and high-risk options $\left(\mathrm{CE}_{\mathrm{DIFF}}\right)$ (subjects are ordered based on their $\mathrm{CE}_{\mathrm{DIFF}}$ as risk-seeking $\leq 0$ and risk-averse $>0$ values $)$. The majority $(\mathrm{N}=14)$ of subjects was riskaverse. 


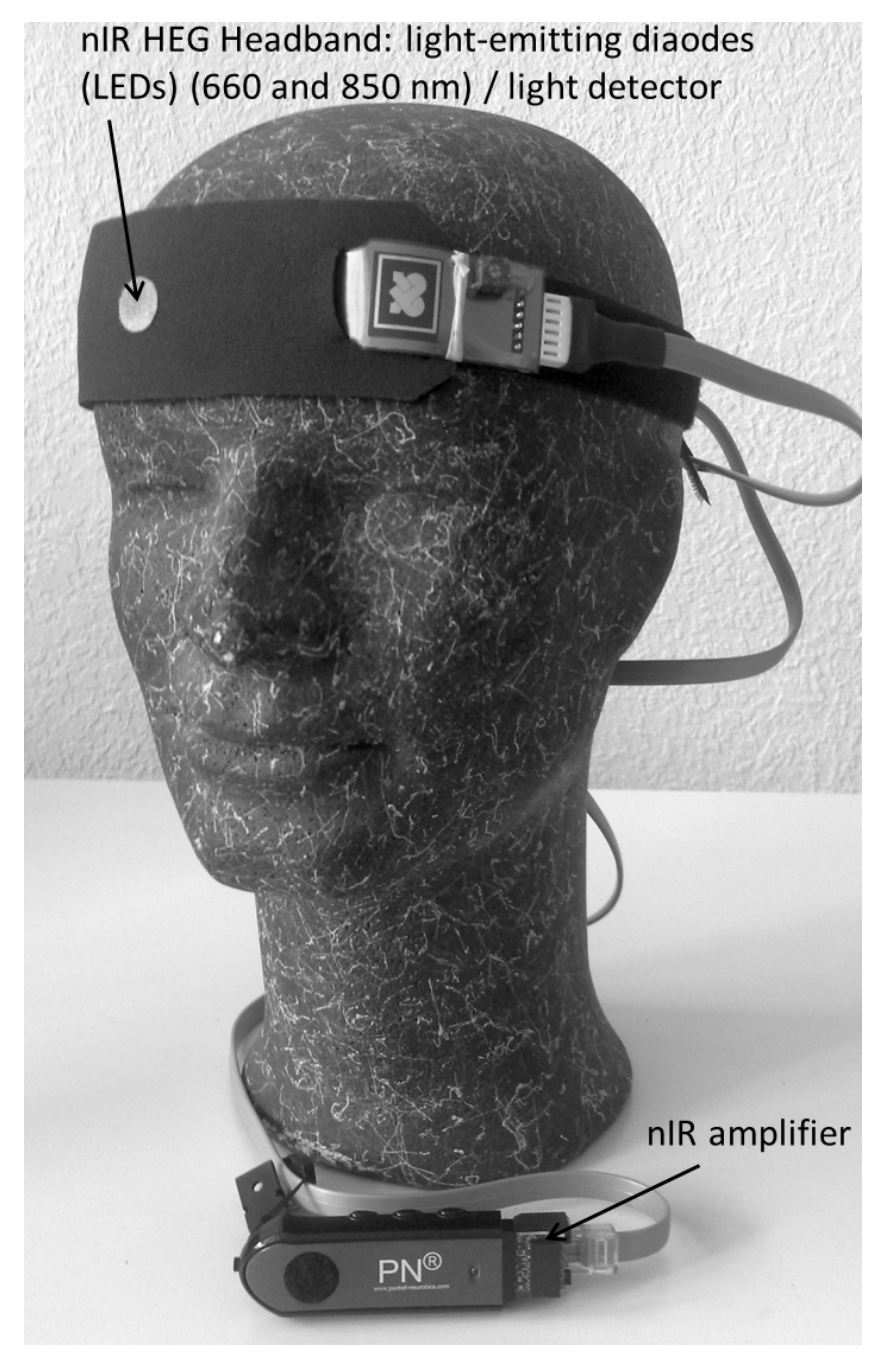

Figure 2. Wireless fNIRS instrument (Biocomp Research Institute, nIR system). The singledistance continuous-wave spectroscopy instrument consists of one channel with one light source with light-emitting diodes (LEDs) (two different wavelengths at $660 \mathrm{~nm}$ and $850 \mathrm{~nm}$ ) and one light detector with a source-detector distance of $3 \mathrm{~cm}$ and a size $90 \times 40 \mathrm{~mm}$. The centre of the sensor was positioned covering F8 according to the international 10-20 system (Jaspers, 1958). 

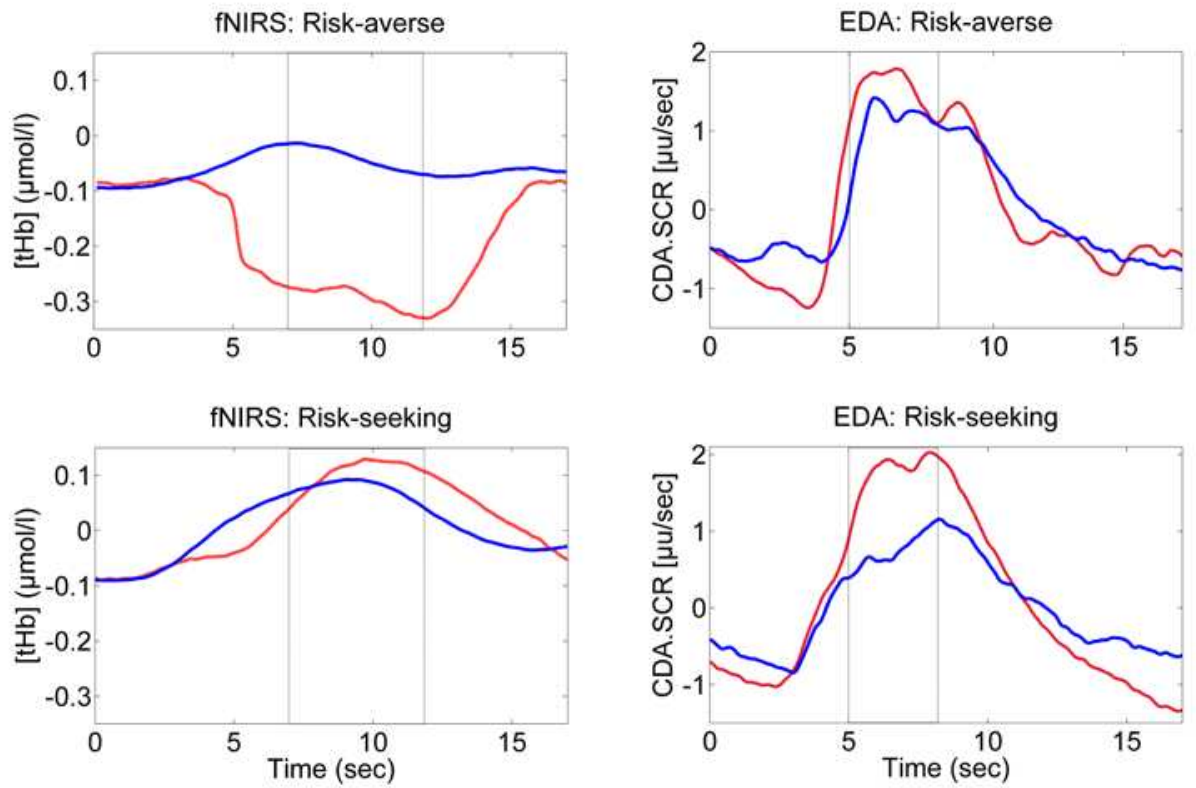

Figure 3. Trial time course of fNIRS and EDA signals. Data are shown from exemplary risk-averse and risk-seeking subjects over the time course of a trial representing (A) [tHb] (fNIRS) and (B) SCRs (EDA) in response to low- (blue) versus high-risk (red) options with the same expected value. Dashed lines indicate trial phases used for analysis. For fNIRS the time interval 7-12 sec after trial onset and for EDA the time interval 5-8 sec after trial onset were used for analysis with 'Expected Value' and 'Risk'. 
(A) fNIRS
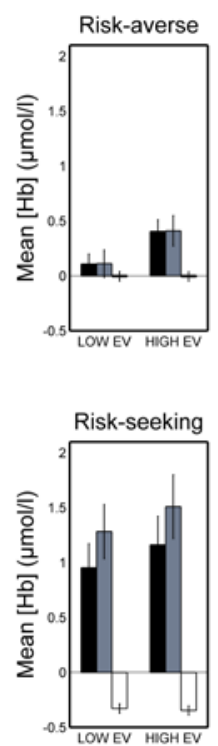

(B) EDA
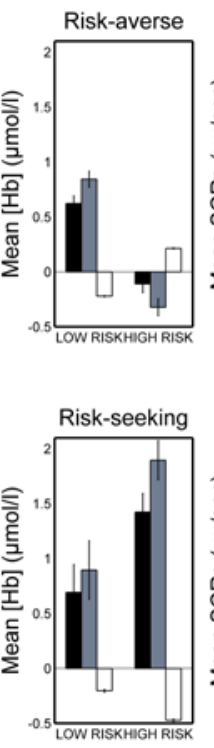
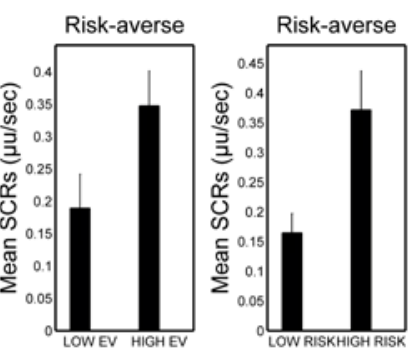

(C) Coherence
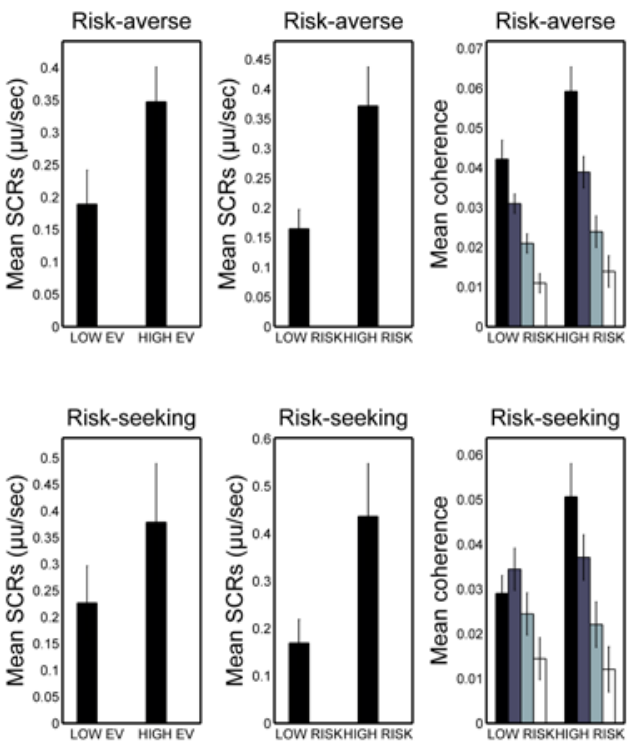

Figure 4. Average responses of fNIRS, EDA: Plots show (A) fNIRS hemodynamic parameter $\Delta[\mathrm{tHb}]$ (black), $\Delta\left[\mathrm{O}_{2} \mathrm{Hb}\right]$ (dark gray) and $\Delta[\mathrm{HHb}]$ (white) (B) EDA derived SCRs (black) for riskaverse and risk-seeking subjects for the factors 'Expected Value' (LOW EV vs. HIGH EV) and 'Risk' (LOW RISK vs. HIGH RISK). Coherence between fNIRS and EDA: Plots show (C) the coherence between $[\mathrm{tHb}]$ and SCRs time series in the heart rate frequency band $(0.7-3 \mathrm{~Hz})$ (black) for the factor 'Risk' (LOW RISK vs. HIGH RISK). For comparison, non-significant changes are shown in the respiration rate $(0.12-0.7 \mathrm{~Hz})$ (dark gray), low frequency oscillations $(0.04-0.12 \mathrm{~Hz})$ (light gray) and very low frequency oscillations $(0.025-0.04 \mathrm{~Hz})$ (white). Error bars represent standard error of the mean (SEM). The corresponding statistical analyses are listed in Table $\mathbf{1 .}$ 
(A) fNIRS

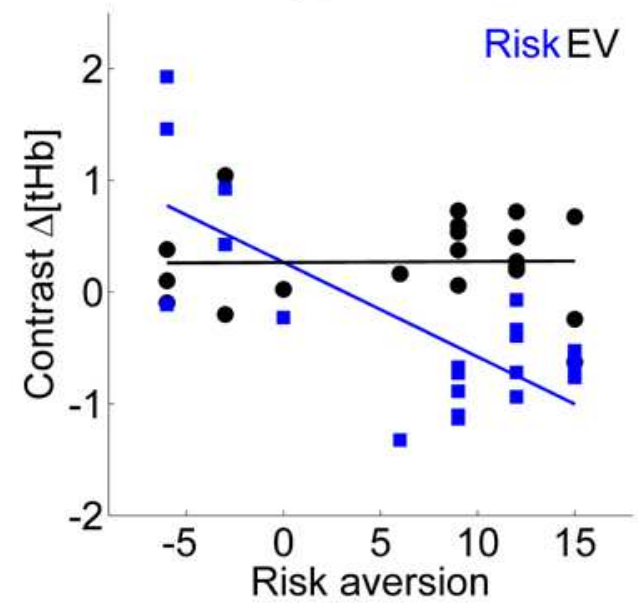

(B) EDA

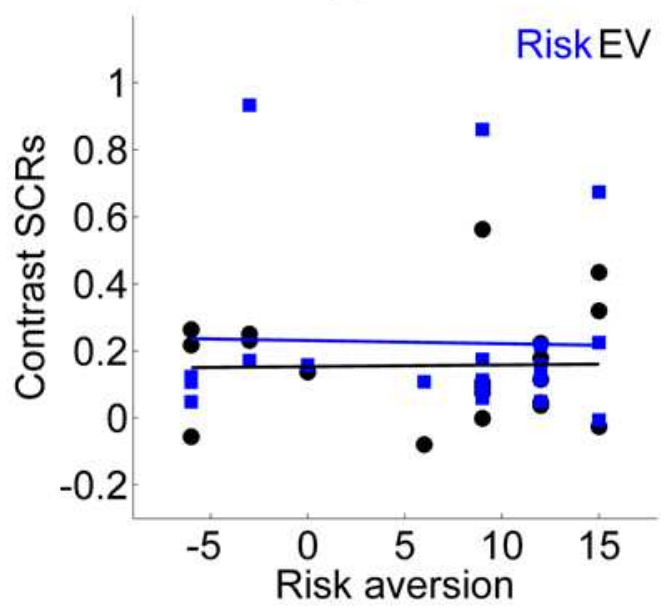

Figure 5. Correlation of risk attitude with fNIRS and EDA data. Plots show correlation between the difference in certainty equivalent $\left(\mathrm{CE}_{\mathrm{DIFF}}\right)$ as index of risk-attitude and the contrasts for $\Delta[\mathrm{tHb}]$ and SCRs of expected value (EV) (black circles) and risk (blue squares). Increasing risk reduced lateral prefrontal cortex activity the more risk-averse subjects were. Conversely, no such effect emerged for EDA and the processing of EV was not affected by risk attitude. 

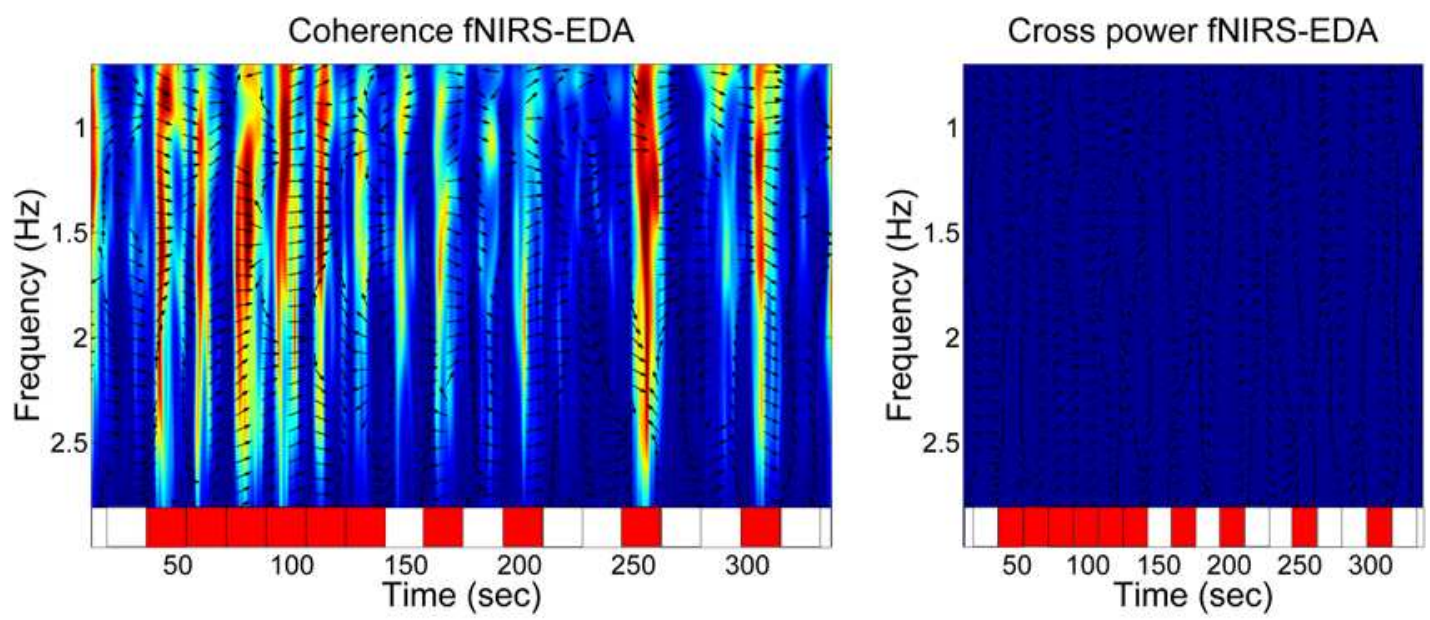

Figure 6. Coherence and cross power between [tHb] and SCRs. Shown is a sample of the colorcoded 2-D coherence map in the frequency band of the heart rate $(0.7-3 \mathrm{~Hz})$ between the $[\mathrm{tHb}]$ and SCRs time series of an example subject over half of the experiment. (Left) Coherence of the two time series illustrating higher coherence values (indicated in red colors) during high-risk trials. Vertical lines indicate the timing of low-risk (white) versus high-risk (red) trials. (Right) Cross power of the two time series; this plot shows that there are no common changes of power in the two time series in the frequency domains, which could have contributed to the coherence increases observed between the two time series. The phase angle between the two time series is indicated by an arrow, but should be interpreted with care: pointing to the right $=$ in-phase or positively correlated, to the left $=$ anti-phase indicates anti-correlation, down $=\mathrm{X}$ leading $\mathrm{Y}$ by $90^{\circ}$, up $=\mathrm{Y}$ leading $\mathrm{X}$ by $90^{\circ}$ (Grinsted et al., 2004). 
Table 1. Average responses of fNIRS and EDA. ANOVA results for $\Delta\left[\mathrm{O}_{2} \mathrm{Hb}\right], \Delta[\mathrm{HHb}], \Delta[\mathrm{tHb}]$, SCRs and the coherence values in the heart rate (HR) frequency band $(0.7-3 \mathrm{~Hz})$ using the fixed factors 'Expected Value' (LOW EV vs. HIGH EV) and 'Risk' (LOW RISK vs. HIGH RISK) for riskaverse and risk-seeking subjects. No interaction effects between the two factors were significant. Listed are F-statistics (F) with degree of freedom (df), partial eta squared $\left(\eta_{\mathrm{p}}{ }^{2}\right)$ and observed post-hoc power $(\mathrm{PHP})$; significant values $(\mathrm{p} \leq 0.05)$ are highlighted $(*)$. The corresponding plots are illustrated in Figure 4.

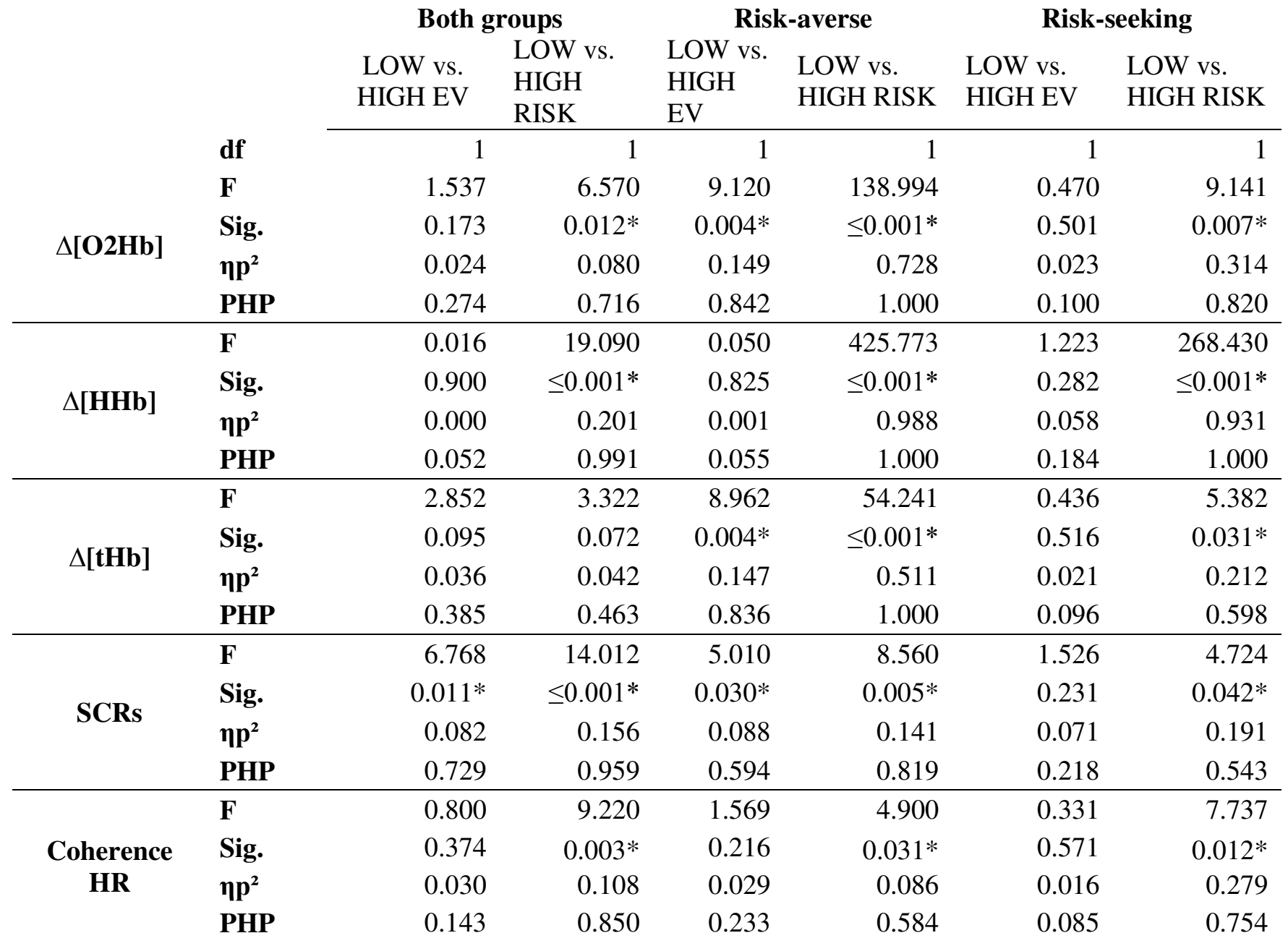


Table 2. Single-subject classification of fNIRS and EDA data. Fisher's linear discriminant analysis is representing the best-performing and cross-validated classification accuracy for each single subject discriminating between low- and high-risk options by keeping expected value (EV) constant. Class discriminatory features were mean $\Delta[\mathrm{tHb}]$ and SCRs amplitudes.

fNIRS: $\Delta[\mathrm{tHb}] \quad$ EDA: SCR

\begin{tabular}{|c|c|c|c|c|}
\hline ID & $\begin{array}{c}\text { LOW } \\
\text { EV }\end{array}$ & $\begin{array}{c}\text { HIGH } \\
\text { EV }\end{array}$ & $\begin{array}{c}\text { LOW } \\
\text { EV }\end{array}$ & $\begin{array}{c}\text { HIGH } \\
\text { EV }\end{array}$ \\
\hline 1 & $60 \%$ & $90 \%$ & $80 \%$ & $70 \%$ \\
\hline 3 & $50 \%$ & $80 \%$ & $90 \%$ & $60 \%$ \\
\hline 4 & $80 \%$ & $60 \%$ & $70 \%$ & $60 \%$ \\
\hline 5 & $60 \%$ & $50 \%$ & $50 \%$ & $50 \%$ \\
\hline 6 & $80 \%$ & $70 \%$ & $70 \%$ & $70 \%$ \\
\hline 7 & $50 \%$ & $60 \%$ & $50 \%$ & $50 \%$ \\
\hline 8 & $90 \%$ & $100 \%$ & $90 \%$ & $90 \%$ \\
\hline 9 & $100 \%$ & $60 \%$ & $80 \%$ & $60 \%$ \\
\hline 13 & $60 \%$ & $70 \%$ & $60 \%$ & $60 \%$ \\
\hline 14 & $88 \%$ & $100 \%$ & $63 \%$ & $67 \%$ \\
\hline 15 & $100 \%$ & $80 \%$ & $70 \%$ & $80 \%$ \\
\hline 17 & $70 \%$ & $70 \%$ & $60 \%$ & $70 \%$ \\
\hline 19 & $80 \%$ & $60 \%$ & $50 \%$ & $60 \%$ \\
\hline 20 & $90 \%$ & $100 \%$ & $50 \%$ & $60 \%$ \\
\hline Total Risk-averse & $75.50 \%$ & $75.00 \%$ & $66.60 \%$ & $64.80 \%$ \\
\hline 2 & $70 \%$ & $60 \%$ & $70 \%$ & $80 \%$ \\
\hline 10 & $80 \%$ & $70 \%$ & $70 \%$ & $70 \%$ \\
\hline 11 & $60 \%$ & $70 \%$ & $70 \%$ & $60 \%$ \\
\hline 12 & $70 \%$ & $60 \%$ & $90 \%$ & $60 \%$ \\
\hline 16 & $80 \%$ & $100 \%$ & $60 \%$ & $60 \%$ \\
\hline 18 & $100 \%$ & $70 \%$ & $80 \%$ & $70 \%$ \\
\hline Total Risk-seeking & $76.70 \%$ & $71.70 \%$ & $73.30 \%$ & $66.70 \%$ \\
\hline Total All & $75.90 \%$ & $\mathbf{7 4 . 0 0 \%}$ & $68.50 \%$ & $65.30 \%$ \\
\hline & \multicolumn{2}{|c|}{ Total NIRS } & \multicolumn{2}{|c|}{ Total EDA } \\
\hline & \multicolumn{2}{|c|}{$73.10 \%$} & \multicolumn{2}{|c|}{$65.30 \%$} \\
\hline
\end{tabular}

